\title{
Accelerating a Network Model of Care: Taking a Social Innovation to Scale
}

\author{
Vickie Cammack and Kerry Byrne
}

\author{
"A social network is like a commonly owned forest: we all stand" \\ to benefit from it, but we also must work together to ensure it \\ remains healthy and productive. This means that social \\ networks require tending, by individuals, by groups and by \\ institutions.
}

Nicholas Christakis and James Fowler

Authors of Connected

\begin{abstract}
Government-funded systems of health and social care are facing enormous fiscal and human-resource challenges. The space for innovation in care is wide open and new disruptive patterns are emerging. These include self-management and personal budgets, participatory and integrated care, supported decision making and a renewed focus on prevention. Taking these disruptive patterns to scale can be accelerated by a technologically enabled shift to a network model of care to co-create the best outcomes for individuals, family caregivers, and health and social care organizations.
\end{abstract}

The connections, relationships, and activities within an individual's personal network lay the foundation for care that health and social care systems/policy must simultaneously support and draw on for positive outcomes. Practical tools, adequate information, and tangible resources are required to coordinate and sustain care.

Tyze Personal Networks is a social venture that uses technology to engage and inform the individual, their personal networks, and their care providers to co-create the best outcomes. In this article, we demonstrate how Tyze contributes to a shift to a network model of care by strengthening our networks and enhancing partnerships between care providers, individuals, and family and friends.

\section{Introduction}

Fiscal austerity, human resource challenges, geographically dispersed family and friends, and women in the workforce all contribute to the considerable pressure on formal care systems. Current approaches to care are generally professionally dominated and models of care typically operate without the active engagement of patients and families. However, our systems of health and social care are dependent upon the freely given care of friends, family, and neighbors. Family caregivers are the backbone of health and social care systems and enable formal care systems, but caregivers often do so at great cost to their own physical, psychological, and financial health (e.g., Schulz et al., 1995; tinyurl.com/73gcjua).
The space for innovation in care is wide open and new disruptive patterns are emerging. These include selfmanagement and personal budgets, participatory and integrated care, supported decision-making, and a renewed focus on prevention. Taking these disruptive patterns to scale can be accelerated by a technologically enabled shift to a network model of care to co-create the best outcomes for individuals, family caregivers, and health and social care organizations. The purpose of this article is to examine how an online tool called Tyze Personal Networks is using technology to scale a social innovation aimed at moving from an individual model of care to a network model of care. In the following sections, a network model of care is described and the outcomes associated with networks are outlined. 


\section{Accelerating a Network Model of Care: Taking a Social Innovation to Scale}

Vickie Cammack and Kerry Byrne

\section{Shifting from an Individual Model of Care to a Network Model of Care}

For the most part, our current approaches to health and social care are dominated by individual models of care that focus on needs and deficiencies. Assessments, diagnosis, prescriptions, and interventions are frequently done as if we live in isolation. In an individual model of care (Figure 1), a person's assets, including their family, friends, and community members are typically not considered or included in care consultations and planning.

A network centric model of care (Figure 2) expands current thinking about health and social care interactions and interventions to include the relationships and connections that individuals have with their friends, family members, community members, caregivers, and care partners. This model recognizes that, to achieve the best outcomes, individuals require communication, problem solving, and collaboration amongst and between informal networks and formal care providers. The systems of support available to people facing life challenges generally span two poles: professional care providers in health and social care settings or groups of family, friends, and neighbours. The latter are comprised of freely given relationships that are first and foremost based on care versus employment and accountability.

\section{Individual Model of Care}

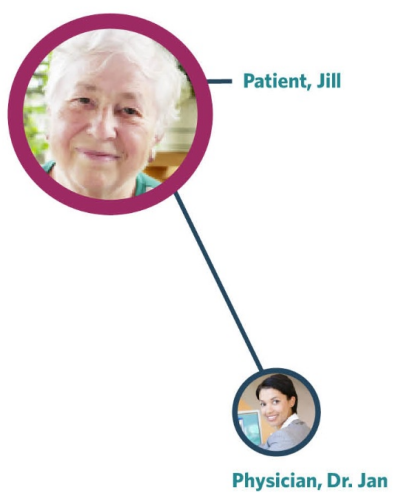

Figure 1. Individual models of care dominate current approaches
Together, these groups constitute an individual's social convoy, that is, the people we count on and the people we draw into our networks during times of need. There is enormous value and wealth inherent in our social convoys. These networks of individuals keep us safe, secure, and healthy by contributing instrumental, informational, and emotional support. The value of our networks is evident through multiple studies demonstrating that people heal more quickly, get sick less often, and use health and human services more efficiently when they have a supportive network (e.g., Umberson and Montez, 2010: tinyurl.com/85fxc5k; Ellaway et al., 1999: tinyurl.com/7x5gp2a). Thus, our networks are key social determinants of our health and ample research reinforces the truth of the proverb "a good friend is the medicine of life".

\section{Network Model of Care}

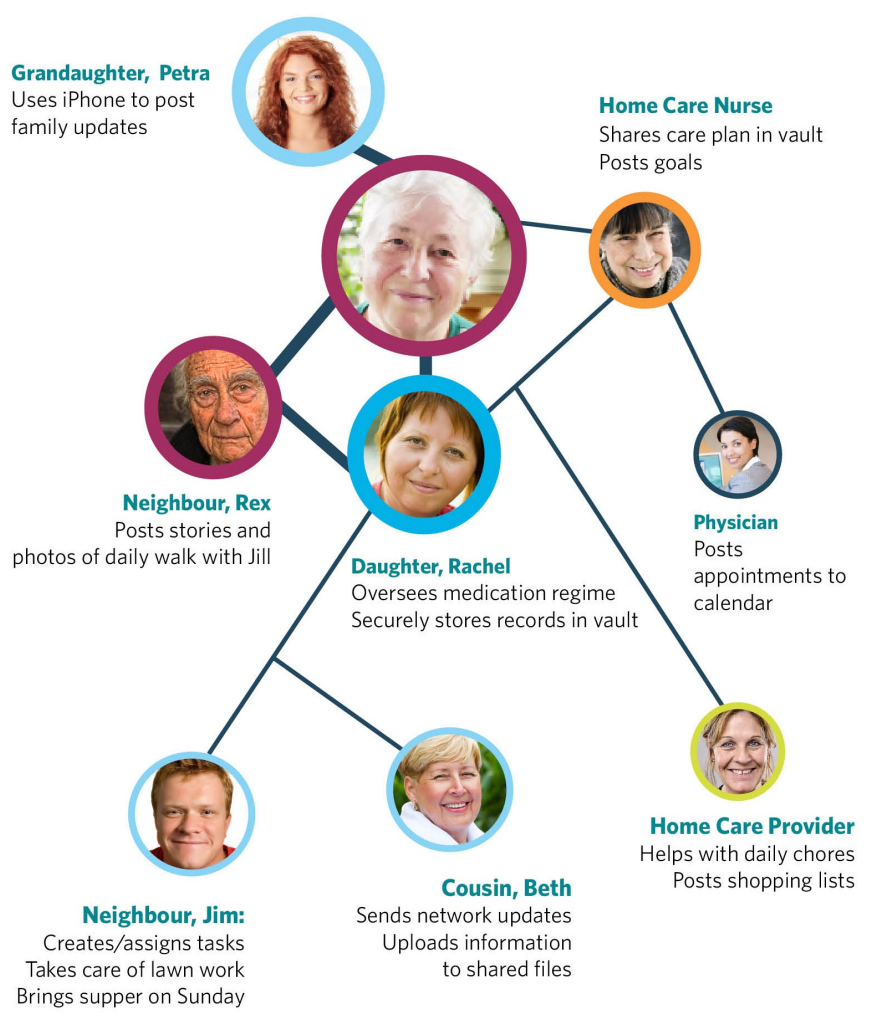

Figure 2. A network model of care includes relationships with friends, family, community members, caregivers, and care providers 


\section{Accelerating a Network Model of Care: Taking a Social Innovation to Scale}

Vickie Cammack and Kerry Byrne

Moreover, the relationships and connections within our networks are the foundation of care that we draw on during times of both celebration and challenge; in particular, we draw on our personal networks. A personal network is a group of individuals who come together to provide intentional and purposeful support for a person(s) facing a life challenge. Notably, a network model of care moves us beyond looking at patients as "units of need" to incorporate the assets individuals possess within their personal networks including what they can do for themselves and what help is available to them. A network model of care also acknowledges that people want to help and care for one another. For example, over three-quarters of Canadians report extending help to family, friends, and neighbors, and 4 in 10 Canadians report being concerned about the needs of others, despite their own life pressures; this represents an increase from the mid 1990s when only 1 in 4 were concerned (Canadian Index of Well-Being, 2011; tinyurl.com/ c3mttw2).

A network model of care builds upon connections between individuals, family, and friends and formal care systems to ensure communication and collaboration between everyone contributing to an individual's well-being. The challenge is to facilitate communication and collaboration between two very different systems of care. Our formal systems operate with a systemic lens of efficiency, effectiveness, expertise, and accountability. In contrast, our family, friends, and neighbours contribute from a lens of love and care - frequently operating with passion, creativity, wisdom, and spontaneity. It is not surprising these two pillars of care struggle to work together. Creating bridges and pathways between these two sytems that empower and enable the contributions of our personal network is a critical element in network centric care.

\section{Tyze Personal Networks: A Social Innovation Journey}

Practical tools, adequate information, and tangible resources are required to harness the power of personal networks of care. Tyze Personal Networks (tyze.com) is a social venture that uses technology to engage, connect, and inform the individual and their personal network members to co-create the best outcomes. Tyze is a social innovation that is using technology as the vehicle for change and was created to contribute to a shift in health and social care from individual to network models of care. Tyze is a technology-enabled service that creates secure, online personal networks to facilitate the interweaving of relationships and connections. It combines expertise in creating resilient personal support with online social networking technologies. Tyze personal networks are created around a specific person or a specific situation. For instance, a network could centre on an older adult who has suffered a stroke, so that care providers and a larger circle of family and friends can provide companionship, monitoring, system navigation, and instrumental support. Access to a Tyze personal network is controlled by the patient/client or close family member, or friend, and network members must be invited to participate.

Tyze has 7000 users and has worked with 50 organizations in Canada, the United States, and the United Kingdom. Tyze partners include: the Province of British Columbia, the Robert Wood Johnson Foundation, the United Kingdom Department of Health, and the JW McConnell Family Foundation.

Tyze was created based on 20 years of experience using community connectors to facilitate personal networks for those who have been marginalized or isolated at Planned Lifetime Advocacy Network (PLAN; plan institute.ca). This experience clearly demonstrated the power and potential of personal networks to change lives and improve health and social outcomes (Etmanski, 2009; tinyurl.com/83co8qg and 2000; tinyurl.com/ 7vvd96w). This core expertise is built into the Tyze software, the networks themselves, and the training materials. Tyze was created to widely distribute and scale PLAN's strategic and proven approach to addressing isolation through the development of personal networks.

Tyze is built on experience and research about networks and the deep rooted knowledge that the lives of anyone experiencing a life challenge - people with disabilities, seniors, and many, many others - can be enriched and transformed by the experience of belonging. This knowledge and experience is embedded in Tyze as we move forward to create tools that can help people to do what they want to do: connect, collaborate, and support one another. The development of Tyze Personal networks is based on several key attributes and values underpinning a network model of care including:

1. A focus on strengthening relationships: Tyze personal networks are small, personal networks of people invested in caring relationships with one another. The interconnections, shared experiences, and motivations 


\section{Accelerating a Network Model of Care: Taking a Social Innovation to Scale}

Vickie Cammack and Kerry Byrne

amongst network members are situated at the core of a network model of care.

2. Interdependence: This is the valued goal versus independence.

3. Asset-based: Families and patients/clients are empowered and engaged through an assumption of competence. For instance, information is shared knowing that patients/clients and family members are able to advocate and use the information to make the best decisions for themselves.

4. Reciprocity and celebration: These elements are central to network sustainability. Features such as photos, stories, and profiles all create opportunities to highlight contributions.

5. Purposeful: Each network has a shared purpose this inspires action and participation.

6. Hospitable: The networks each have a coordinator or host who extends invitations, welcomes members and their contributions, and generates activity in a network.

7. Focus on contribution by all: Tyze personal networks make it easy for everyone to pitch in because they are "in the know" about specific needs and can contribute in a way that is convenient or meaningful for them.

8. Bridge to the formal system: Access to information and expertise assists personal networks to provide the best support possible. Tyze features such as the Vault and File drop provide safe ways to share confidential information. Paid professionals can easily participate for short periods of time, for example in acute episodes, and then be removed from the network.

In summary, a Tyze personal network is a space to build, grow, and mobilize our personal networks. It is, by design, a positive, welcoming, and warm space to come together and support one another.

\section{Insights}

Through partnerships with various health and social care organizations implementing Tyze, we have identified several challenges and opportunities for using technology to scale a network model of care, as detailed in a report by the Robert Wood Johnson Foundation (2011; tinyurl.com/blm52bp). For instance, in some cases, individuals that are most in need of a network model of care do not have access to, or are not comfortable with, technology. For these reasons, second-degree Internet access - the engagement of persons who remain offline through loved ones who are online - is an increasingly important concept (Fox, 2010; tinyurl.com/2b5w3k5). It means that people who are not able to use computers (for a variety of reasons including disability or fear of computers) are still "online", and thus accessing the benefits of being online, because of a relative who goes online with or for them.

As well, the bridge between individuals, family, and friends and formal care systems is a difficult one to build - there are privacy concerns and time and resource constraints. A key barrier to the expansion of a network model of care is that it requires shared communication and meaningful collaboration, across time and place, to achieve high-quality care. People from different sectors, backgrounds, and motivations must come together and collaborate to enhance care and support. Tyze personal networks function as hubs of communication including updates, messages, files, requests and offers of help, and resource sharing - all within a space that is private and secure. Clarity around the value proposition for everyone involved in a network model of care is critical. Improved care may be the key to "what's in it for me" for some, while for others it is the social return on investment that is valued. Moreover, while patient engagement and the inclusion of family caregivers in healthcare teams has consistently been identified in multiple care situations, modern day healthcare systems struggle to do this in a meaningful way.

Network participation, that is, continued engagement and active involvement of members of the network, is both a challenge and an opportunity. Networks take time to create and nurture and require contributions from members to be sustained. Using technology opens up new ways of contributing for long-distance caregivers, as an example. Often times, people want to lend a hand to a care situation or a person's life but are not sure what to do or how to do it. A network member may have a specific time they can pitch in or a special skill that can be incorporated into care, but without the interactions facilitated on the network, no one would "make the match" between what is available and the opportunity to contribute. Also, participation across generations, that is, intergenerational networks, ensure that everyone can contribute. 


\section{Accelerating a Network Model of Care: Taking a Social Innovation to Scale}

Vickie Cammack and Kerry Byrne

Engaging in an online personal network makes it easier for people to know how to pitch in. As part of a mixed methods program of research at Tyze, including quarterly online surveys and qualitative interviews with our users, we found that Tyze strengthens connections and mobilizes support (see Cammack et al., 2012 P03.3; tinyurl.com/85ggcgo). For example, $90 \%$ of respondents to a recent Tyze user survey reported that their Tyze network helps them to share information and $70 \%$ reported that Tyze helped them contribute to a care task.

Finally, we have learned a great deal about what it takes to implement a new technology in health and social care settings. Perhaps the most important lesson is identifying social intrapreneurs - individuals who work inside organizations and develop, promote, and advocate for solutions such as Tyze. These individuals are willing to embrace novelty and the challenges that go along with it, because they are able to see past the barriers and are willing to work in partnership to develop innovations. They are also willing to pursue these innovations based on their unique knowledge of the values, goals, and workflows of their organizations and the people they serve. In our case, working closely with intrapreneurs has resulted in much success and learning - and close partnerships based on shared goals and a vision for better outcomes through a network model of care.

\section{Conclusion}

The economic, health, and social climate is ripe for a culture shift to a network model of care. Technology holds a great deal of promise to address some of the challenges, however, adoption of new models can be slow and scaling these models beyond pilot and earlystage testing is challenging. Building bridges between individuals, family, and friends and formal systems is historically difficult, with or without the use of a technology. Time and resources are often cited as barriers to communication and collaboration with family members and patients.

To effectively achieve a network model of care, multiple and disparate parts of health and social care support systems need to be connected. However, creating a value proposition that resonates for all network members (e.g., formal care providers, paid and unpaid network members, health and non-health) requires skilled communication and practical solutions given limited resources and time pressures. Tyze Personal Networks has worked through several of these challenges and contributed to a shift to a network model of care by applying 20 years of experience building and sustaining personal networks; developing key partnerships with health and social care organizations and social intrapreneurs; and creating a research program focused on understanding and demonstrating the value of online personal networks.

\section{About the Authors}

Vickie Cammack is President and CEO of Tyze Personal Networks. In this role, Vickie focuses her attention and expertise on how best to deliver online, personal support networks to people facing life challenges. Vickie is also a co-founder of Planned Lifetime Advocacy Network (PLAN), a pioneer social enterprise supporting families to secure the future of their family member with a disability. She created PLAN's Personal Network program, a unique response to the isolation experienced by people with disabilities and mentored the spread of PLAN groups in 40 locations globally. Vickie is the recipient of the Meritorious Service Medal of Canada, the Canadian Psychological Association's Humanitarian Award, and Simon Fraser University President's Club Distinguished Community Leadership Award. She co-led a Canadian exploration on sustainability and social innovation and is a Fellow with Social Innovation Generation.

Kerry Byrne, $\mathrm{PhD}$, is the Director of Research at Tyze Personal Networks. She has over 10 years of healthcare research experience and cares deeply about improving care for families. Through her work, she strives to give a voice to families' and patients' experiences with health and social care and improve the mobilization of formal and informal support for family caregivers and persons experiencing life challenges. Her areas of research expertise are in family caregiving, care transitions, home care, and relationship-centered care.

Citation: Cammack, V. and K. Byrne, M. 2012.

Accelerating a Network Model of Care: Taking a Social Innovation to Scale. Technology Innovation

Management Review. July 2012: 26-30. 